EESTI NSV TEADUSTE AKADEEMIA TOIMETISED. XIII KÖIDE FOOSIKA-MATEMAATIKA- JA TEHNIKATEADUSTE SEERIA. 1964, NR. 2

ИЗВЕСТИЯ АКАДЕМИИ НАУК ЭСТОНСКОИ ССР. ТОМ ХІІ СЕРИЯ ФИЗИКО-МАТЕМАТИЧЕСКИХ И ТЕХНИЧЕСКИХ НАУК. 1964, ㄱ 2

\title{
О НАИЛУЧШИХ КВАДРАТУРНЫХ ФОРМУЛАХ С ФИКСИРОВАННЫМИ УЗЛАМИ
}

\author{
М. ЛЕВИН, \\ кандидат физико-математических наук
}

На практике для заключения о точности приближенного интегрирования часто сравниваются приближенные значения интеграла, вычисленные по $n$ и по $2 n$ узлам. Если модуль разности этих значений меньше допускаемой ошибки, то за величину интеграла принимается второе значение.

В настоящей заметке показывается, что при сравнении экстремальных ошибок (точных верхних граней ошибок, получаемых при вычислениях по заданной формуле для функций заданного множества) второе приближенное значение интеграла в некоторых случаях достаточно вычислять по $n+1$ или $n+2$ узлам.

1. Для множества $W_{0}^{(1)} L^{(2)}$ функций $f(x)$, на отрезке $[0,1]$ абсолютно непрерывных и удовлетворяющих условиям $f(0)=0$, $\left\|f^{\prime}(x)\right\|_{L^{(s)}} \leqslant M$, рассмотрим квадратурные формулы

$$
\int_{0}^{1} f(x) d x=\sum_{k=0}^{n-1} p_{k} f\left(x_{k}\right)+r_{n}{ }^{(0)}(f)
$$

и

$$
\int_{0}^{1} f(x) d x=\sum_{k=0}^{n-1} p_{k} f\left(x_{k}\right)+\sum_{k=0}^{r-1} B_{k} f\left(y_{k}\right)+R_{n}(0)(f),
$$

где $x_{k}, p_{k}(k=0,1, \ldots, n-1), y_{j}(j=0,1, \ldots, r-1)$ - заданные числа.

Пусть

$$
r_{n}{ }^{(0)}=\sup _{f \in W_{0}^{(1)} L^{(2)}}\left|r_{n}^{(0)}(f)\right|, \quad R_{n}^{(0)}=\sup _{f \in W_{0}^{(1)} L^{(2)}}\left|R_{n}{ }^{(0)}(f)\right|:
$$

Требуется для рассматриваемого множества функций построить наилучшую формулу (2), т. е. найти значения $B_{k}(k=0,1, \ldots, r-1)$ так, чтобы величина $R_{n}{ }^{(0)}$ была наименьшей.

a) Пусть формула (1) наилучшая для функций множества $W_{0}^{(1)} L^{(2)}$. Тогда $\left[{ }^{1}\right]$

$$
\begin{gathered}
p_{k}=\frac{2}{2 n+1}, \quad x_{k}=\frac{2 k+2}{2 n+1} \quad(k=0,1, \ldots, n-1) \\
r_{n}{ }^{(0)}=\frac{M}{(2 n+1) \sqrt{3}} .
\end{gathered}
$$


Используя равенство

$$
f(x)=\int_{0}^{1} f^{\prime}(t) E(x-t) d t
$$

где

$$
E(x)= \begin{cases}0, & x \leqslant 0 \\ 1, & x>0\end{cases}
$$

получаем, применяя неравенство Буняковского, по (2)

$$
R_{n}{ }^{(0)}=M\left\{\int_{0}^{1} K^{2}(t) d t\right\}^{1 / 2},
$$

где

$$
K(t)=1-t-\sum_{k=0}^{n-1} p_{k} E\left(x_{k}-t\right)-\sum_{k=0}^{r-1} B_{k} E\left(y_{k}-t\right) .
$$

Фиксируя следующие параметры

$$
r=n, \quad y_{k}=0,5\left(x_{k-1}+x_{k}\right) \quad(k=0,1, \ldots, n-1), x_{-1}=0,
$$

сводим задачу к минимизации интеграла

$$
I=\int_{0}^{1} K^{2}(t) d t
$$

Для отыскания значений $B_{k}(k=0,1, \ldots, n-1)$, минимизирующих этот интеграл, имеем систему уравнений $I_{B_{l}}^{\prime}=0 \quad(l=0,1, \ldots, n-1)$ (решение этой системы действительно дает минимум интегралу (7), так как все определители $\left|I_{B_{i}{ }_{j}}\right|_{i, j=0}^{l}$ при $l=0,1, \ldots, n-1$ положительны), которую можно записать в виде

$$
\begin{aligned}
& \sum_{k=0}^{n-1} B_{k} \int_{0}^{1} E\left(y_{k}-t\right) E\left(y_{l}-t\right) d t=\int_{0}^{1}(1-t) E\left(y_{l}-t\right) d t- \\
& -\sum_{k=0}^{n-1} p_{k} \int_{0}^{1} E\left(x_{k}-t\right) E\left(y_{l}-t\right) d t \quad(l=0,1, \ldots, n-1),
\end{aligned}
$$

откуда

$$
\begin{gathered}
\sum_{k=0}^{l} B_{k} y_{k}+y_{l} \sum_{k=l+1}^{n-1} B_{k}=y_{l}-\frac{y_{l}^{2}}{2}-\sum_{k=0}^{l-1} p_{k} x_{k}-y_{l} \sum_{k=l}^{n-1} p_{k} \\
(l=0,1, \ldots, n-1) .
\end{gathered}
$$

Подставляя сюда значения (6) и (3), получаем

$$
\sum_{k=0}^{l}(2 k+1) B_{k}+(2 l+1) \sum_{k=t+1}^{n-1} B_{k}=\frac{1}{2(2 n+1)} \quad(l=0,1, \ldots, n-1)
$$


и, значит,

$$
B_{0}=\frac{1}{2(2 n+1)}, \quad B_{1}=B_{2}=\ldots=B_{n-1}=0
$$

Таким образом, для множества $W_{0}^{(1)} L^{(2)}$ наилучшая формула (2) с фиксированными значениями (6), получаемая удлинением наилучшей формулы (1), имеет вид

$$
\int_{0}^{1} f(x) d x=\frac{2}{2 n+1} \sum_{k=0}^{n-1} f\left(\frac{2 k+2}{2 n+1}\right)+\frac{1}{2(2 n+1)} f\left(\frac{1}{2 n+1}\right)+R_{n}{ }^{(0)}(f)
$$

По (5), учитывая равенство (4), получаем для этой формулы

$$
R_{n}{ }^{(0)}=\frac{M}{(2 n+1) \sqrt{3}} \sqrt{1-\frac{3}{4(2 n+1)}}=r_{n}{ }^{(0)} \sqrt{1-\frac{3}{4(2 n+1)}} .
$$

6) Пусть $n>1$ - нечетно и (1) есть формула Симпсона, для которой, как можно подсчитать,

$$
r_{n}{ }^{(0)}=\frac{M}{3(n-1)} \sqrt{5-\frac{8}{n-1}} .
$$

Тогда аналогичными рассуждениями получаем, что для $W_{0}{ }^{(1)} L^{(2)}$ наилучшая формула (2), получаемая удлинением формулы Симпсона (1) путем добавления новых узлов, лежащих по средине между узлами формулы (1), имеет вид

$$
\begin{aligned}
& \int_{0}^{1} f(x) d x=\frac{1}{3(n-1)}\left\{f(1)+4\left[f\left(\frac{1}{n-1}\right)+f\left(\frac{3}{n-1}\right)+\ldots+f\left(\frac{n-2}{n-1}\right)\right]+\right. \\
& \left.\quad+2\left[f\left(\frac{2}{n-1}\right)+f\left(\frac{4}{n-1}\right)+\ldots+f\left(\frac{n-3}{n-1}\right)\right]+\frac{1}{4} f\left(\frac{1}{2 n-2}\right)\right\}+R_{n}^{(0)}(f)
\end{aligned}
$$

для которой

$$
R_{n}{ }^{(0)}=\frac{M}{3(n-1)} \sqrt{5-\frac{273}{32(n-1)}} .
$$

2. Пусть $W^{(1)} L^{(2)}$ множество функций $f(x)$, на $[0,1]$ абсолютно непрерывных и удовлетворяющих условию $\left\|f^{\prime}(x)\right\|_{L^{(2)}} \leqslant M$.

Для этого множества функций средн формул внда

$$
\int_{0}^{1} f(x) d x=\sum_{k=0}^{n-1} p_{k} f\left(x_{k}\right)+q f(0)+\sum_{k=0}^{n-1} B_{k} f\left(y_{k}\right)+R_{n}(f)
$$

прн заданных $p_{k}, x_{k}, y_{k}$ будем искать наилучшую формулу.

Справедливо очевидное неравенство

$$
\sup _{f \in W^{(1)} L^{(2)}}\left|R_{n}(f)\right| \geqslant \sup _{f \in W_{0}^{(1)} L^{(2)}}\left|R_{n}(f)\right| .
$$


Пусть

$$
q=1-\sum_{k=0}^{n-1}\left(B_{k}+p_{k}\right)
$$

тогда

$$
R(c)=0, \quad c=\text { const. }
$$

Используя это, имеем

$$
R_{n}[f(x)]=R_{n}[f(x)-f(0)]
$$

поэтому

$$
\sup _{f \in W^{(1)} L^{(2)}}\left|R_{n}(f)\right| \leqslant \sup _{f \in W_{0}^{(1)} L^{(2)}}\left|R_{n}(f)\right| .
$$

откуда по (9) получаем

$$
\sup _{f \in W^{(1)} L^{(2)}}\left|R_{n}(f)\right|=\sup _{f \in W_{0}^{(1)} L^{(2)}}\left|R_{n}(f)\right| .
$$

Отсюда и из пункта 1 следует, что наилучшей для $W^{(1)} L^{(2)}$ формулой (8) является:

$$
\begin{aligned}
& \text { a) формула } \\
& \int_{0}^{1} f(x) d x=\frac{2}{2 n+1} \sum_{k=0}^{n-1} f\left(\frac{2 k+2}{2 n+1}\right)+\frac{1}{2(2 n+1)}\left[f(0)+f\left(\frac{1}{2 n+1}\right)\right]+R_{n}(f) \text {, }
\end{aligned}
$$

где

$$
\sup _{f \in W^{(1)} L^{(2)}}\left|R_{n}(f)\right|=\frac{M}{(2 n+1) \sqrt{3}} \sqrt{1-\frac{3}{4(2 n+1)}}
$$

а за формулу (1) взята наилучшая для $W^{(1)} L^{(2)}$ формула

$$
\int_{0}^{1} f(x) d x \approx \frac{2}{2 n+1} \sum_{k=0}^{n-1} f\left(\frac{2 k+2}{2 n+1}\right)+\frac{1}{2 n+1} f(0)
$$

и $y_{k}=0,5\left(x_{k-1}+x_{k}\right)$;

б) Формула

$$
\begin{gathered}
\int_{0}^{1} f(x) d x=\frac{1}{3(n-1)}\left\{f(1)+4\left[f\left(\frac{1}{n-1}\right)+f\left(\frac{3}{n-1}\right)+\ldots+f\left(\frac{n-2}{n-1}\right)\right]+\right. \\
\left.+2\left[f\left(\frac{2}{n-1}\right)+f\left(\frac{4}{n-1}\right)+\ldots+f\left(\frac{n-3}{n-1}\right)\right]+\frac{1}{4}\left[3 f(0)+f\left(\frac{1}{2 n-2}\right)\right]\right\}+R_{n}(f)
\end{gathered}
$$

где

$$
\sup _{f \in W^{(1)} L^{(2)}}\left|R_{n}(f)\right|=\frac{M}{3(n-1)} \sqrt{5-\frac{273}{32(n-1)}}
$$


а за (1) взята формула Симпсона и за новые узлы $y_{k}$ - середины отрезков $\left[x_{k}, x_{k+1}\right]$.

ЛИ ТЕ Р А Т У Р А

1. Дорон и н Г. Я. $\mathrm{K}$ вопросу о формулах механических квадратур. Сб. трудов Днепропетровского инж.-стр. ин-та, 1955, № $1-2$, стр. $210-217$.

2. Никольский С. М. Квадратурные формулы. М., 1958.

Институт кибернетики

Академии наук Эстонской ССР
Поступила в редакцию

24. X 1963

\title{
PARIMATEST FIKSEERITUD SÕLMPUNKTIDEGA KVADRATUURVALEMITEST
}

\author{
M. Levin, \\ füüsika-matemaatikateaduste kandidaat
}

Resümee

Artiklis tuletatakse funktsioonide hulga $W_{0}(1) L^{(2)}$ jaoks parim valem (2) tingimuse (6) puhul, kusjuures valem (1) loetakse antuks ja parimaks selle funktsioonide hulga jaoks. Analoogiline ülesanne lahendatakse juhul, kui (1) on Simpsoni valem. Samad ülesanded lahendatakse ka funktsioonide hulga $W\left({ }^{(1)} L^{(2)}\right.$ jaoks.

Eesti NSV Teaduste Akadeemia Küberneetika Instituut
Saabus toimetusse

24. X 1963

\section{ON THE BEST QUADRATURE FORMULAE WITH FIXED POINTS}

\section{Levin}

\section{Summary}

In this article the best formula (2) is constructed satisfying the condition (6) for the class of functions $W_{0}^{(1)} L^{(2)}$. The formula (1) is assumed to be given and the best for this class of functions. An analogic problem is solved for (1) being Simpson's formula. These problems are solved for the class of functions $W^{(1)} L^{(2)}$, too.

Academy of Sciences of the Estonian S. S. R., Institute of Cybernetics
Received

Oct. 24th, 1963 